\title{
Passivation of cobalt nanocluster assembled thin films with hydrogen
}

\author{
C.P. Romero ${ }^{\text {a,* }}$, A. Volodin ${ }^{\text {a }}$, M. Di Vece ${ }^{b}$, H. Paddubrouskaya ${ }^{\text {a }}$, Huan Wang ${ }^{c}$, A. Vantomme ${ }^{\text {c }}$, \\ C. Van Haesendonck ${ }^{\mathrm{a}}$, P. Lievens ${ }^{\mathrm{a}, * *}$ \\ a Laboratory of Solid State Physics and Magnetism, Department of Physics and Astronomy, KU Leuven, Celestijnenlaan 200d, box 2414, BE-3001 Leuven, Belgium \\ ${ }^{b}$ Center for Individual Nanoparticle Functionality (CINF), Department of Physics, Technical University of Denmark, DK-2800 Kgs. Lyngby, Denmark \\ c Institute of Nuclear and Radiation Physics, Department of Physics and Astronomy, KU Leuven, Celestijnenlaan 200d, box 2418, BE-3001 Leuven, Belgium
}

\section{A R T I C L E I N F O}

Article history:

Received 21 June 2011

Received in revised form 8 April 2012

Accepted 12 April 2012

Available online 20 April 2012

\section{Keywords:}

Clusters

Hydrogen

Passivation

Cobalt

Cobalt oxide

Magnetic force microscopy

Rutherford backscattering spectrometry

\begin{abstract}
A B S T R A C T
The effect of hydrogen passivation on bare and Pd capped cobalt nanocluster assembled thin films was studied with Rutherford backscattering spectrometry (RBS) and magnetic force microscopy (MFM) after exposure to ambient conditions. The nanoclusters are produced in a laser vaporization cluster source in which the helium carrier gas was mixed with hydrogen. RBS revealed that oxidation of the Co nanoclusters is considerably reduced by the presence of hydrogen during cluster formation. The capping did not modify the influence of the passivation. The hydrogen passivation method is especially effective in cases when capping of the films is not desirable, for example for magnetic studies. Clear differences in the magnetic domain structures between hydrogen passivated and non-passivated Co nanocluster films were demonstrated by MFM and are attributed to a difference in inter-cluster magnetic interaction, which is critically depending on the $\mathrm{CoO}$ content. The hydrogen passivation method may be used to tailor and stabilize properties of nanocluster assembled thin films.
\end{abstract}

(c) 2012 Elsevier B.V. All rights reserved.
The fascinating properties of nanoclusters extend over a wide range of topics that include optics, plasmonics, catalysis, electronics, and magnetism [1]. For example, cobalt silicide nanostructures/nanowires are potentially useful for electronic devices because of the material's particularly good electrical conductance [2]. Ferromagnetic cobalt nanocluster films represent a promising alternative for high-density and highspeed magnetic information storage technology and even hyperthermia cancer treatment in the case of functionalized nanoparticles [3]. Accurate magnetic field control is possible in multiferroic magnetoelectric composite nanostructures and particulate nanocomposite films [4,5], making nanoclusters a very relevant subject, both from fundamental and applications point of view. However, the oxygen concentration in such nanosized materials is a crucial parameter. Considering for example the surface to volume ratio, the presence of small amounts of oxygen can have a significant impact on the nanostructure characteristics. Partial or complete oxidation may inhibit or even destroy the relevant functionalities under study. In order to prevent oxidation, samples often are covered with capping material to avoid contact with the ambient [6]. For example, nanocluster films of lead can be capped with silicon to protect the superconducting properties [7].

Although the presence of a capping layer has proven to be effective against oxidation, the capping layer itself may interfere with

\footnotetext{
* Corresponding author.

** Corresponding author. Tel.: + 3216327207.

E-mail addresses: christian.romero@fys.kuleuven.be (C.P. Romero), peter.lievens@fys.kuleuven.be (P. Lievens).
}

the functional properties. Chemical reduction by for example hydrogen plasma is also employed for certain materials. Ideally, nanoclusters should be passivated before exposure to the ambient, hence preventing post growth oxidation. Passivation by hydrogen can be used to reduce the concentration of surface defects in Si nanocrystals, but can have a strong influence on the overall electronic properties [8]. Typically, hydrogen is binding to the dangling bonds caused by the high defect density on the surface. The inability of cobalt to absorb (diffuse) hydrogen under ambient conditions makes it a good candidate to investigate the passivating properties of attached hydrogen [9].

In this work we introduce hydrogen in the carrier gas of a laser vaporization cluster source during the production of cobalt nanoclusters. By observing the time-of-flight (TOF) mass spectra of our gas phase cluster production we know that two separate cluster growth regimes are established: according to the addition or not of the $\mathrm{H}_{2}$. We then study the hydrogen passivation effect for bare and $\mathrm{Pd}$ capped cobalt nanoclusters by measuring the oxygen content with Rutherford backscattering spectrometry (RBS) after exposure to the ambient. The magnetic domain patterns of the non-covered cobalt nanocluster films are examined with magnetic force microscopy (MFM), providing a further confirmation of the passivation effect.

Gas phase cluster sources [10] have the benefit of producing welldefined individual nanoclusters. Such a device can produce nanoclusters with sizes ranging from a few to thousands of atoms [11,12]. Here, cobalt nanoclusters were produced in a laser vaporization source [10]. Vaporized atoms condensate in a pulsed high pressure 
of typically $\sim 700 \mathrm{kPa}$ pressure and the resulting nanoclusters are extracted into a beam directed towards the deposition chamber. For this purpose noble gasses are typically used to avoid chemical interaction with the cluster species. Two cluster growth regimes were employed in our study: one with a pure He flow and one with a mixed flow (98\% He and $2 \% \mathrm{H}_{2}$ with purity $>99.95 \%$ ).

Cluster assembled films were grown by deposition in a low energy $(<0.4 \mathrm{eV} /$ atom $)$ regime at room temperature in an ultra high vacuum deposition chamber with pressure of $\sim 10^{-7} \mathrm{~Pa}\left(10^{-9} \mathrm{mbar}\right)$. A detailed description of this setup can be found elsewhere [13]. The cluster size distribution was monitored with TOF mass spectrometry. The stability of the Co cluster samples was investigated with RBS, after storage under ambient conditions for several days and then also after 3 months; we refer to the oxidation levels in our samples. Although the exposure to ambient certainly results in an exposure to oxygen, it cannot be ruled out that traces of oxygen were already present on the substrate and this oxygen migrated from the substrate into the cluster assembled films. In some cases the oxygen need to be reduced in a mild atmosphere of $\mathrm{H}_{2}$ [14]. Two samples (A and B) were produced on polished (0001) oriented sapphire and capped with $3.5 \mathrm{~nm}$ of Pd using a commercial e-beam evaporator loaded with a pure Pd rod (purity 99.99\%). The Pd capping layer should minimize post production oxidation. Additionally, two cobalt nanocluster samples ( $\mathrm{C}$ and $\mathrm{D}$ ) were prepared on a $\mathrm{SiO}_{2}$ substrate without a capping layer. This enables us to compare the oxygen content in capped and uncapped samples. The thickness of the samples $C$ and D was increased by a factor of 5 in an attempt to protect the lower layers of the sample from oxidation by the oxidized top layer. Samples A and C were produced in the high-purity He gas flow (purity 99.9999\%), while samples B and D were produced in the gas mixture of $\mathrm{He}$ with $2 \% \mathrm{H}_{2}$ (see Table 1 ).

In Fig. 1a a TOF mass spectrum is presented starting from mass zero. The spectrum is the average of 1800 separate measurements. The graph clearly confirms the presence of $\mathrm{H}_{2}, \mathrm{He}, \mathrm{Co}$, and $\mathrm{Co}+\mathrm{H}$ in the accumulated transient (the averaged measurements). A Co $+\mathrm{H}$ shoulder at the base of the Co peak (inset of Fig. 1a) can be clearly distinguished for the sample that was produced with the hydrogen containing gas. The two other spectra (red and green color in Fig. 1a) were recorded in the absence of hydrogen, one recorded immediately before and one recorded after the hydrogen containing spectrum. These spectra only show a Co peak without a $\mathrm{Co}+\mathrm{H}$ shoulder. We conclude that hydrogen is absorbed on atomic Co. We infer from the low intensity of the $\mathrm{Co}+\mathrm{H}$ peak that most of the Co is present in the beam in the form of pure Co.

The mass spectra are detected with a microchannel plate (MCP), and therefore the indicated values are in arbitrary units. Even though only a relative comparison is possible, we present this TOF spectrum (Fig. 1a) because of the excellent signal/noise ratio that allows to deduce the ratio between the peak areas as $\mathrm{Co}: \mathrm{CoH} \approx(9 \pm 2): 1$. Thus the fraction of $\mathrm{CoH}$ ranges from $9 \%$ to $15 \%$ when compared to the Co peak.

Table 1

Details of the investigated cluster assembled Co films. Column named "sample description" details the gasses used to fabricate the samples. In the column "Number of atoms per unit area" we display the actual counts as measured by RBS in units of $1 \times 10^{17}$ atoms $/ \mathrm{cm}^{2}$. In this same column we present in square brackets the corresponding thickness in bulk-like thin films. In parentheses we present measured thickness with atomic force microscopy. The last column displays the atomic \% of oxygen of each sample.

\begin{tabular}{lllll}
\hline Sample & $\begin{array}{l}\text { Sample } \\
\text { description }\end{array}$ & $\begin{array}{l}\text { Number of atoms per unit area } \\
\left(1 \times 10^{17} \text { atoms } / \mathrm{cm}^{2}\right)\end{array}$ & $\begin{array}{l}\text { Co cluster layer } \\
\text { (\% oxygen })\end{array}$ \\
\cline { 3 - 4 } & & Co & Pd & \\
\hline $\mathrm{A}$ & $\mathrm{He}$ & $1.3[14 \mathrm{~nm}]$ & $0.3[4 \mathrm{~nm}]$ & $22 \pm 7$ \\
$\mathrm{~B}$ & $\mathrm{He}+\mathrm{H}\{98 \%+2 \%\}$ & $1.8[19 \mathrm{~nm}]$ & $0.5[7 \mathrm{~nm}]$ & $10 \pm 9$ \\
$\mathrm{C}$ & $\mathrm{He}$ & $6.7[72 \mathrm{~nm}](49 \mathrm{~nm})$ & $\mathrm{N} / \mathrm{A}$ & $37.5 \pm 5$ \\
$\mathrm{D}$ & $\mathrm{He}+\mathrm{H}\{98 \%+2 \%\}$ & $5.0[54 \mathrm{~nm}](41 \mathrm{~nm})$ & $\mathrm{N} / \mathrm{A}$ & $<10$ \\
\hline
\end{tabular}

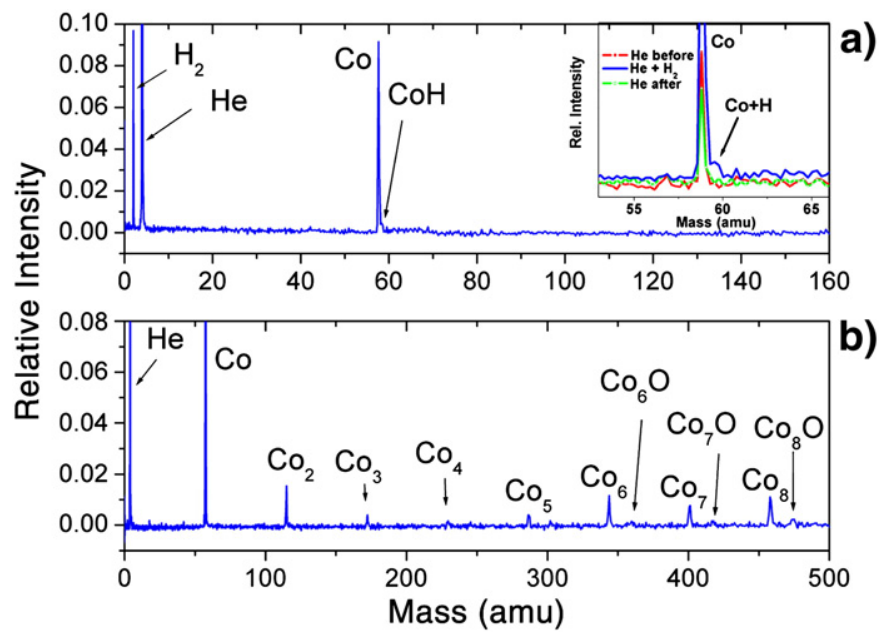

Fig. 1. (a) Time-of-flight mass spectra of the beam produced with the $\mathrm{He}$ and $\mathrm{H}_{2}$ gas mixture. The inset provides a closer view of the $\mathrm{Co}$ and $\mathrm{CoH}$ peaks. The results demonstrate the attachment of a $\mathrm{H}$ atom to the Co monomer. (b) Time-of-flight mass spectra of the beam produced with pure He.

This clearly indicates that $\mathrm{CoH}$ is not produced preferentially. If we assume that only $12 \%$ of the Co atoms have attached hydrogen, as can be estimated from the area of the peaks, this would imply that if all this hydrogen was at the surface of a cluster consisting of 400 Co atoms (mean value of the cluster production see Fig. 2a and b) it would have about 48 hydrogen atoms on the surface. If we further assume that the clusters are spherical we get a fraction of surface atoms between $40 \%$ and $50 \%$, that is, $160-200$ atoms. On the other hand, Fig. 1b shows several peaks in a wider mass range as well as Co oxide. Fig. 1 clearly demonstrates the different gas phase cluster production regimes used to prepare our samples. The next paragraph details further.

The cluster formation depends on pressure, temperature, and the ablation materials being used. At certain conditions, i.e. in a specific growth regime, all the small clusters are used up to produce larger ones. In the case of $\mathrm{Co}$ with $\mathrm{H}_{2}+\mathrm{He}$ we clearly see that, apart from the monomer, small species of a few Co atoms are not present, but there is an abundance of larger clusters as shown in Fig. 2(a). This means that all the small sized clusters take part in the process of

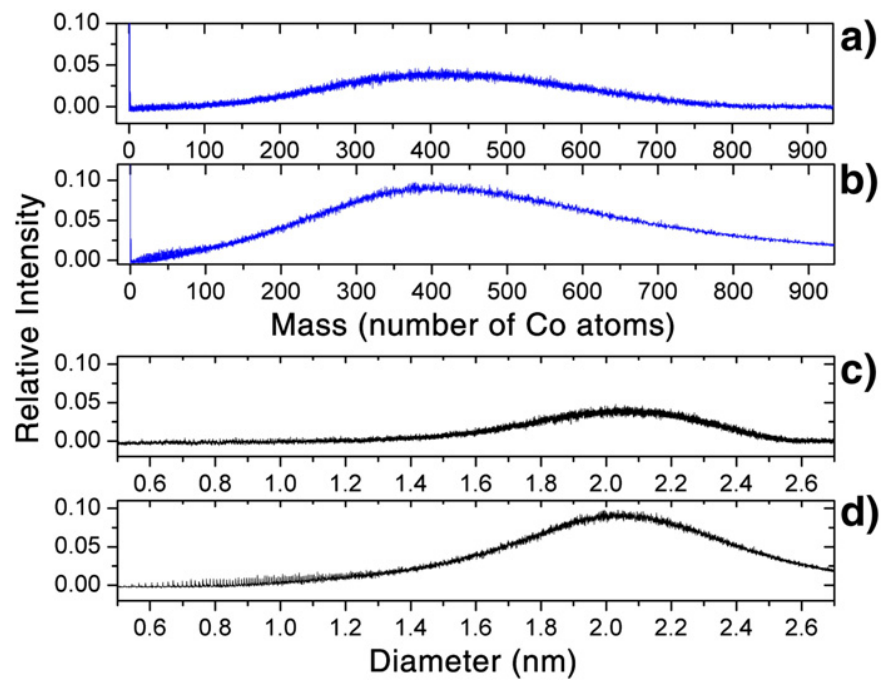

Fig. 2. (a) and (b) show the time-of-flight mass spectra of the cluster beam as produced with the He and $\mathrm{H}_{2}$ gas mix, and with pure He, respectively. (c) and (d) show the cluster distribution with respect to diameter for clusters produced in $\mathrm{He}$ with $\mathrm{H}_{2}$ and in $\mathrm{He}$ without $\mathrm{H}_{2}$ respectively. 
producing larger clusters. This is why we only observe one peak in Fig. 1a. In the case of Co with only He gas the growth regime is different resulting in the detection of $\mathrm{CO}_{2}$ or $\mathrm{CO}_{3}$, for example. Thus the differences as apparent in Figs. 1 and 2 are interpreted as evidence for two different growth regimes.

One important issue is the timing of exposure of the Co atoms to hydrogen. Following the ablation of Co atoms by the laser pulse, the vaporized material is exposed to very high concentrations of gas, resulting in condensation to cobalt clusters in the expansion volume; here the initial gas pulse is at $900 \mathrm{kPa}$ for $200 \mu \mathrm{s}$. At this instance the ratio of gas molecules versus $\mathrm{Co}$ atoms is highest. The average pressure in this chamber (source chamber) is $7 \times 10^{-3}-2 \times 10^{-3}$ Pa during working cycles. In the deposition chamber where the clusters are landed on the substrate the pressure is $5 \times 10^{-6}-1 \times 10^{-5}$ Pa during working cycles.

This pressure gradient is managed through 3 steps of differential pumping. If we further consider that there is only $2 \%$ of $\mathrm{H}_{2}$ compared to $98 \% \mathrm{He}$, we have a partial pressure of $\mathrm{H}_{2}$ of $5 \times 10^{-8}-1 \times 10^{-7} \mathrm{~Pa}$ in the deposition stage. If we consider the sticking probability of a $\mathrm{H}_{2}$ molecule to be 1 when it hits the surface then the time it takes is well over $100 \mathrm{~min}$ to cover the whole surface with one layer of $\mathrm{H}_{2}$ molecules. All the samples were fabricated in less than $30 \mathrm{~min}$. With these considerations we conclude that the hydrogen passivation occurs at initial stages of the cluster production, i.e. during formation, and not post-deposition.

In Fig. 2a and b a TOF mass spectrum of Co nanoclusters is shown with $\mathrm{H}_{2}$ and without $\mathrm{H}_{2}$, respectively. The mass is presented in number of Co atoms. Assuming a spherical geometry for our clusters and using a Wigner-Seitz radius for cobalt of $0.1379 \mathrm{~nm}$ [15], we can transform the mass in our spectra into a cluster diameter (in nm, see Fig. 2c and d). The size of the produced Co nanoclusters varies between $1.5 \mathrm{~nm}$ and $2.5 \mathrm{~nm}$ for clusters produced in the presence of $\mathrm{H}_{2}$ (Fig. 2c) and from 1.1 to $2.8 \mathrm{~nm}$ for clusters produced in the absence of $\mathrm{H}_{2}$ (Fig. 2d). The slightly broader size range can be attributed to the presence of Co oxides that promote the overall clustering process. As is clear from Fig. 2, for both production regimes the average size is very similar with the peak maximum observed around $2.1 \mathrm{~nm}$.

Rutherford backscattering spectrometry (RBS) was employed to measure the chemical compositions of the cluster assembled films. In Fig. 3 we present the experimental and simulated spectra for sample $C$. The inset shows the experimental geometry used in the backscattering measurements. The arrows labeled with $\mathrm{O}$ at surface, $\mathrm{Co}$, $\mathrm{O}$ in $\mathrm{SiO}_{2}$, and $\mathrm{Si}$ in $\mathrm{SiO}_{2}$ indicate the energy of $\mathrm{He}^{+}$ions scattered from the $\mathrm{O}$ and $\mathrm{Co}$ atoms at the sample surface, and from the $\mathrm{O}$ and $\mathrm{Si}$ atoms at the interface between the Co cluster layer and $\mathrm{SiO}_{2}$ substrate, respectively. A simulation of the random spectrum given by

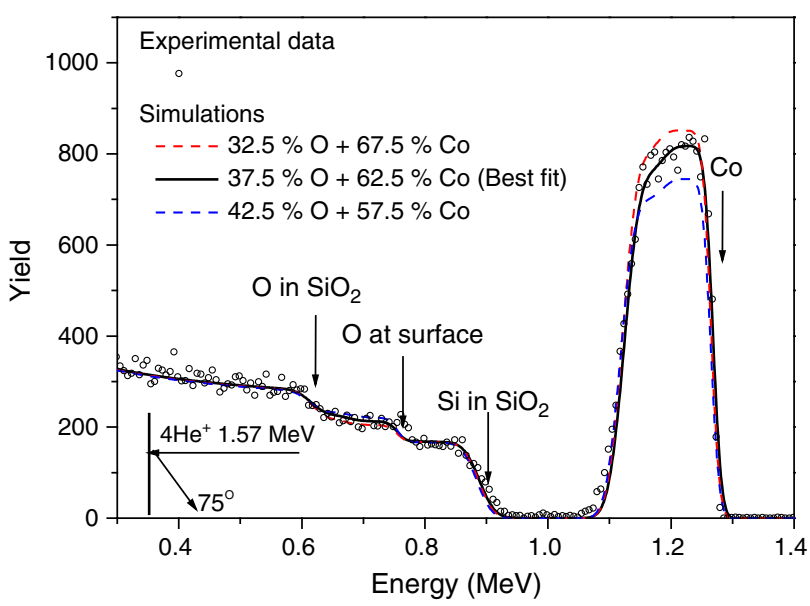

Fig. 3. Experimental data (open circles) and simulated data (solid lines) for the RBS measurements on sample $\mathrm{C}$. the RUMP program (solid line) [16] reveals that the content of Co and $\mathrm{O}$ in the layer are $62.5 \%$ and $37.5 \%$, respectively, which give the best fit to the experimental data. Since RBS is sensitive to a heavy element such as Co, just a $5 \%$ increase/decrease of the Co content (with respect to $62.5 \%$ ) in the cluster film will result in a significant deviation between the experimental data and the simulations (see the dashed lines in Fig. 3). Hence, the error for the 0 content in sample C can be estimated to be less than $5 \%$. It can be seen in the last column of Table 1 that the concentration of the oxygen is significantly lower in both the Pd covered and the bare Co cluster samples with the Co clusters produced in a $2 \% \mathrm{H}_{2}$ mixture. In addition, the $\mathrm{Co}$ and $\mathrm{Pd}$ (for sample A and sample B) coverage in atoms $/ \mathrm{cm}^{2}$ can also be inferred from the RBS spectra (see Table 1). Due to the unknown atomic density of the cluster films, it is not possible to extract the thickness of the layers from the RBS results. In order to provide an intuitive feeling for the total amount of Co or Pd atoms in our samples, the converted corresponding thickness of bulk-like thin films is given between square brackets in Table 1 . The thickness of samples $C$ and D was also measured with atomic force microscopy (AFM) and the obtained values were about $15 \mathrm{~nm}$ lower, as indicated by the numbers between parentheses in Table 1.

After 10 days of storage under ambient conditions RBS reveals an oxygen content as given in Table 1, in the column labeled "Co cluster layer (\% Oxygen)". Both Co and Pd are partially oxidized, up to around $37.5 \%$ for the Co nanoclusters produced in He (samples A and C). The amount of oxygen for the Co nanoclusters produced in the $2 \% \mathrm{H}_{2}$ mixture is, however, significantly lower, both for the Pd covered sample and for the bare Co cluster sample. It cannot be excluded that the Pd layer thickness plays a role in the oxidation process, however, considering the degree of oxidation its effect is not strong. The Co nanoclusters in sample B are only $10 \%$ oxidized and for sample D a value of less than $10 \%$ is found. This is consistent with the strong passivating nature of hydrogen on the cobalt nanoclusters. This property is highly desirable from a practical point of view, especially in the cases when capping of the films should be avoided.

The magnetic properties of the Co cluster films are sensitive to their chemical composition, in particular the relative amount of the CoO component [17]. Therefore, it is very important to be able to stabilize the thin film systems and to eliminate the influence of the environment. Typically, stabilization is achieved using different coatings. However, in many cases the capping layers alter the magnetic properties of the systems and are therefore undesirable. With the aim of better understanding the influence of the hydrogenation procedure on the magnetic properties we characterized samples $C$ and D with magnetic force microscopy (MFM). The dependence of magnetism on the amount of $\mathrm{CoO}$ allows us to relate the different oxygen content to the magnetic interaction between clusters. This way we are able to confirm the results of the RBS data.

MFM measurements were carried out with a Dimension 3100 scanning probe microscope (Veeco Instruments Inc.) operated in the tapping lift mode using commercial MFM probes (Nanosensors) with tips magnetized along the tip axis. The MFM images were taken in the demagnetized state at zero external field. MFM is a suitable technique for the visualization of magnetic surface domains and can provide valuable information about the strength of magnetic interactions between nanoclusters $[18,19]$. Since MFM senses the vertical component of the force gradient between the sample and the tip, it is sensitive to the strength and polarity of near-surface stray fields produced by ferromagnetic samples. This allows obtaining information about the overall magnetic domain structure from an MFM image. By comparing the MFM image with the corresponding AFM image correlations are established between the domain structure and the topographic data. We only probed the uncovered samples (C and D) with MFM. The reason for measuring only the uncapped samples is that Pd, as an almost magnetic material, may induce interferences with the Co layer [20]. 
In Fig. 4(a) an MFM image of the domain structure of Co cluster sample $C$ is presented, while the corresponding topographic AFM image is presented in Fig. 4(b). Distinctive magnetic domains are present as areas of yellow and blue contrast with typical dimension around $1 \mu \mathrm{m}$. These films have a perpendicular out-of-plane magnetic anisotropy, while the fact that magnetic domains are considerably larger than a single particle confirms the existence of magnetic interactions between Co clusters. The MFM image of the H-treated Cocluster film (sample D), which is presented in Fig. 4(c), reveals the presence of considerably larger domains (around $3 \mu \mathrm{m}$ ). Moreover, a "feather-like" magnetic pattern associated with these larger-size magnetic domains is observed, which is distinctively different from the "granular" domain structure in sample $C$. This difference in magnetic appearance can be attributed to a difference in strength of the magnetic interactions between the nanocluster aggregates. The large $\mathrm{CoO}$ content reduces the exchange interaction between clusters. Therefore, the magnetic domain size of the non-hydrogenated cluster film is smaller than that of the hydrogenated one. On the other hand, the stronger magnetic interactions in the H-containing Co-cluster film can be explained by the presence of more ferromagnetic cobalt metal, leading to strong dipolar interactions [12,21]. This may be accounted for by the fact that the adsorbed hydrogen prevents oxidation and hence is responsible for a larger concentration of cobalt metal. The weaker interaction (= smaller domains) in sample $\mathrm{C}$ is the result of a high concentration of cobalt oxide which is non-magnetic at room temperature. A study of the magnetic properties of a thin Co film as a function of oxidation has indeed demonstrated that the magnetic moment per Co atom decreases considerably after oxidation [22]. The amount of oxygen in cobalt cluster assembled films determines its ferromagnetic properties as demonstrated by investigations of Co cluster assembled films with different degrees of oxidation [15,23,24].
Strictly speaking, at this point it is unclear whether the weaker magnetic interaction of the oxidized sample $(\mathrm{C})$ is caused by a reduced cobalt metal content or alternatively that an oxide shell reduces the magnetic coupling between the cobalt cores [25]. In our investigation we focused on the influence of the passivation.

As illustrated by the AFM image of sample D in Fig. 4(d), the observed granular structure typically shows fairly large spherical-like grains with sizes of the order of a few tens of nanometer, with an average value around $30 \mathrm{~nm}$ diameter. This image clearly confirms the result of earlier studies that upon deposition the clusters as produced in the beams ( 1.1 to $2.8 \mathrm{~nm}$ diameter, see Fig. $2 \mathrm{~d}$ ) tend to coagulate into larger nanoclusters. This image confirms the spherical shape of deposited Co cluster aggregates with a much larger size when compared to the size inferred from the TOF mass spectrum (see Fig. 2c and $\mathrm{d}$ ). The images reveal an average surface roughness around $3 \mathrm{~nm}$ for all of the produced cluster films, both for the bare Cocluster film (sample C) and the H-treated Co-cluster film (sample D). However, the magnetic domain structure is not markedly influenced by the surface topography.

MFM measurements carried out after 15 days do not reveal any changes in the magnetic domain configuration for sample $\mathrm{D}$. On the other hand, some degraded regions were observed in the domain pattern of sample $C$. This confirms the effectiveness of our hydrogen passivation method. After 3 months, samples C and D still present significant differences. We emphasize that the MFM results reveal a strong correlation with the RBS data. This indicates we are dealing with a strong and enduring passivation effect.

In conclusion we have demonstrated that the addition of $2 \% \mathrm{H}_{2}$ gas in the He carrier gas flow of a cluster source has a profound effect on the amount of oxidation of deposited cobalt nanoclusters, that is, on the final outcome of the "as deposited" thin films. The presence of

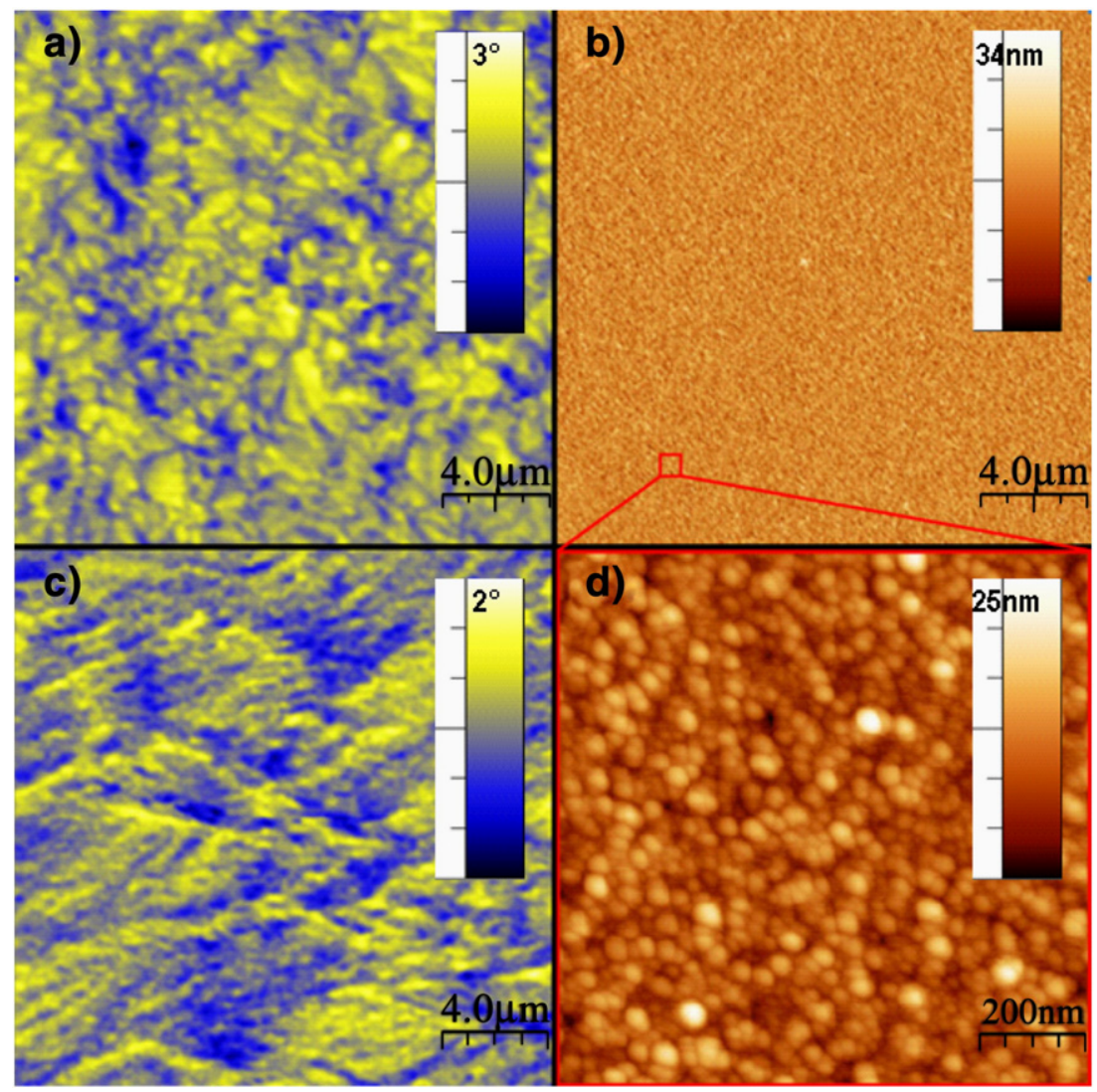

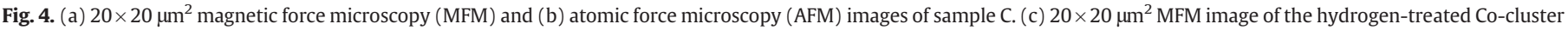

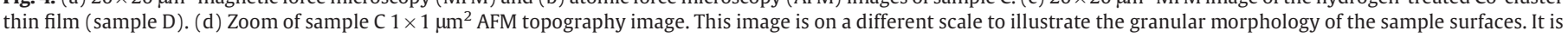
representative for both samples presented in this figure. 
hydrogen results in 20\% less oxidation of the Co clusters with a Pd capping layer. In the case of Co clusters without Pd this reduction exceeds 50\%. The effect of the Pd on the Co cluster layer is probably related to the attraction of the atomic hydrogen to the Pd layer, thus leaving the Co cluster available for oxidation, although the exact chemical pathway still needs to be determined. We conclude from the analysis of the TOF mass spectra that the most probable mechanism for the low level of oxygen is the influence of adsorbed hydrogen on the Co clusters, protecting them against oxidation. We estimated that not all of the atoms at the surface of the clusters have attached hydrogen, but even with this limited coverage we obtain significantly different physical properties of the cluster assembled films.

One important question remains unanswered, how does the $\mathrm{H}$ retain its passivation effect during the process of cluster agglomeration on the substrate? The exact mechanism is beyond the scope of this study and would require another study. We can only point out that agglomeration does occur, that is, smaller clusters form larger ones. The role of hydrogen in this process is unclear. We know that hydrogen remains in the agglomerated clusters and we claim that its effects are present as the samples show different properties that are correlated with the absence of oxygen. In accordance to this, the MFM measurements on the bare cobalt nanocluster films confirm the passivation effect of hydrogen by the appearance of a modified magnetic domain structure (in particular the observation of larger domains) due to a larger ferromagnetic cobalt metal content. Oxidized cobalt nanoclusters have a weaker interaction between clusters. Our results demonstrate that the hydrogen passivation method is especially effective in cases where capping of the films is not desirable. Our passivation method can be used to control the amount of oxidation in a nanocluster assembled thin film of any composition that is produced by a gas aggregation cluster source.

\section{Acknowledgment}

This research is supported by the Research Foundation - Flanders (FWO) as well as by the Belgian Interuniversity Attraction Poles (IAP) and the Flemish Concerted Action (GOA) research programs.

\section{References}

[1] L. Canali, Ph.D. thesis, Delft University of Technology, The Netherlands, ISBN:906464-78-79, 2000.

[2] C.I. Tsai, P.H. Yeh, C.Y. Wang, H.W. Wu, U.S. Chen, M.Y. Lu, W.W. Wu, L.J. Chen, Z.L Wang, Cryst. Growth Des. 9 (10) (2009) 4514

[3] R. Hergt, S. Dutz, R. Muller, M. Zeisberger, J. Phys. Condens. Matter 18 (2006) S2919.

[4] Y. Wang, J. Hu, Y. Lin, C.W. Nan, NPG Asia Mater. PY 2 (2) (2010) 61.

[5] V.K. Valev, M. Di Vece, M.J. Van Bael, D. Grandjean, S. Decoster, A. Vantomme, T. Verbiest, P. Lievens, J. Appl. Phys. 105 (2009) 114907.

[6] J.N. Huiberts, R. Griessen, J.H. Rector, R.J. Wijngaarden, J.P. Dekker, D.G. de Groot, N.J. Koeman, Nature 380 (1996) 231.

[7] J. Cuppens, C.P. Romero, P. Lievens, M.J. Van Bael, Phys. Rev. B 81 (2010) 064517.

[8] S. Cheylan, R.G. Elliman, Appl. Phys. Lett. 78 (9) (26 Feb. 2001).

[9] C.P. Romero, J.I. Avila, E. Cisternas, G.B. Cabrera, A.L. Cabrera, K. Temst, M.J. Van Bael, J. Mater. Sci. 42 (2007) 18.

[10] W. Bouwen, P. Thoen, F. Vanhoutte, S. Bouckaert, F. Despa, H. Weidele, R.E. Silverans, P. Lievens, Rev. Sci. Instrum. 71 (2000) 54.

[11] C. Binns, Curr. Opin. Solid State Mater. Sci. 8 (2004) 203.

[12] P.G. Reinhard, E. Suraud, Wiley-VCH Verlag GmbH, ISBN: 3-527-40345-0, 2004 pp. 40.

[13] N. Vandamme, E. Janssens, F. Vanhoutte, P. Lievens, C. Van Haesendonck, J. Phys. Condens. Matter 15 (2003) S2983.

[14] C.P. Romero, J.I. Avila, R.A. Trabol, H. Wang, A. Vantomme, M.J. Van Bael, P. Lievens, A.L. Cabrera, Int. J. Hydrog. Energy 35 (2010) 2262.

[15] V.L. Moruzzi, P.M. Marcus, K. Schwarz, P. Mohn, J. Magn. Magn. Mater. 54-57 (1986) 955.

[16] Rutherford Backscattering Spectroscopy analysis package. Built on Genplot, This Package Provides Comprehensive Analysis and Simulation of RBS and ERD Spectra, Computer Graphic Service, Ltd., 1996

[17] R. Morel, A. Brenac, C. Portemont, J. Appl. Phys. 95 (7) (2004) 3757.

[18] V.F. Puntes, P. Gorostiza, D.M. Aruguete, N.G. Bastus, A.P. Alivisatos, Nat. Mater. 3 (2004) 263.

[19] A.N. Dobrynin, M.J. Van Bael, K. Temst, P. Lievens, New J. Phys. 9 (2007) 258.

[20] J. Flouquet, O. Taurian, J. Sanchez, M. Chapellier, J.L. Tholence, Phys. Rev. Lett. 38 (1977) 2.

[21] S. Rives, A. Catherinot, F. Dumas-Bouchiat, C. Champeaux, A. Videcoq, R. Ferrando, Phys. Rev. B 77 (2008) 085407.

[22] L. Smardz, U. Kobler, W. Zinn, J. Appl. Phys. 71 (1992) 5199.

[23] X.Z. Li, X.H. Wei, R. Skomski, D.J. Sellmyer, J. Nanopart. Res. 12 (2010) 789.

[24] A.N. Dobrynin, D.N. Ievlev, C. Hendrich, K. Temst, P. Lievens, U. Hörmann, J. Verbeeck, G. Van Tendeloo, A. Vantomme, Phys. Rev. B 73 (2006) 245416.

[25] D. Srikala, V.N. Singh, A. Banerjee, B.R. Mehta, S. Patnaik, J. Phys. Chem. C 112 (2008) 13882. 